\title{
Fehlgeschlagene Osteosynthesen des Tibiakopfes
}

\author{
Thomas Braun, Andreas Wentzensen
}

\section{Zusammenfassung}

Der Bruch der Schienbeinkopfgelenkfläche als ein Last tragendes Gelenk stellt eine Herausforderung für die Wiederherstellung dar. Die Osteosynthese soll das Rekonstruktionsergebnis halten sowie eine ausreichende Stabilität für die Bruchheilung gewährleisten. Kann dies Ziel nicht erreicht werden, sind gegebenenfalls frühe Reosteosynthese, Verfahrenswechsel oder eine zusätzliche osteosynthetische Versorgung bis hin zu späteren korrigierenden Eingriffen erforderlich.

\section{Einleitung}

Der Bruch des Schienbeinkopfes stellt aufgrund von anatomischen und verletzungstypischen Besonderheiten als ein Last tragendes Gelenk eine spezielle Herausforderung für die operative Versorgung dar. Ziel ist es, die Gelenkfläche des Kniegelenkes möglichst anatomisch zu rekonstruieren und Beinachsenabweichungen zu vermeiden. Die Aufgabe der Osteosynthese besteht darin, das Rekonstruktionsergebnis nach offener oder geschlossener Reposition zu halten und eine Übungsstabilität im Kniegelenk zu ermöglichen. Eingesetzt werden hierzu unterschiedliche Schrauben und Plattensysteme wie auch der Fixateur externe.

Für die Osteosynthese der Gelenkfraktur (Tibiakopf) gilt das Prinzip der Gelenkflächenrekonstruktion und Übungsstabilität (siehe auch Bsp.1).

Die Hauptprobleme, denen sich der Operateur bei der Durchführung der Osteo-

OP-JOURNAL 2004; 20: 218-221

(c) Georg Thieme Verlag KG Stuttgart · New York synthese des Tibiakopfes stellen muss, sind der Ausgleich von Inkongruenzen der Gelenkfläche und Achsenabweichungen des Tibiaplateaus in der Horizontalebene.

Von einer fehlgeschlagenen Osteosynthese kann man dann sprechen, wenn ein von der Frakturgeometrie abhängiges gutes Repositions- oder Rekonstruktionsergebnis intraoperativ nicht erreicht oder nicht ausreichend stabilisiert werden kann.

\section{Das Prinzip der}

Gelenkflächenrekonstruktion und Übungsstabilität

\section{Beispiel 1}

44-jähriger Patient mit einer vollständig artikulären mehrfragmentären medialen und lateralen Fraktur des Tibiakopfes (C3.3). Intraoperativ konnte ein gutes Rekonstruktionsergebnis mit dem Ausgleich von Inkongruenzen des Tibiaplateaus mit regelrechter horizontaler Ausrichtung erreicht werden (Abb.1). Die fehlende Kooperation des Patienten führte durch die Vollbelastung des Beines postoperativ zum Korrekturverlust (Abb.2). Sofort nach Feststellung der Dislokation der Fragmente, 9 Tage nach der Primäroperation, wurde eine erneute Reposition der Fraktur und Wechsel der nur proximal winkelstabil besetzten L-Platte auf eine vollständig winkelstabile LCP durchgeführt (Abb. 3). Das Repositionsergebnis wurde intraoperativ mit einer dreidimensionalen Röntgenrekonstruktion (ISO C3D) kontrolliert (Abb. 4). Durch die erneute frühzeitige Vollbelastung des Beines durch den Patienten kam es nach der zweiten Operation zur erneuten Dislokation der Fragmente. Ein erneuter Eingriff erschien daraufhin bei völlig fehlender Compliance nicht mehr sinnvoll.

Keine Osteosynthese des Tibiakopfes erlaubt eine Vollbelastung des Beines in den ersten 6 Wochen.

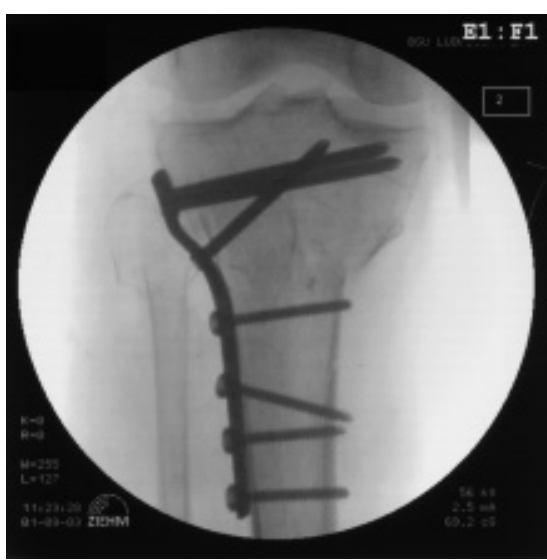

Abb.1 Röntgenbild Querformat.

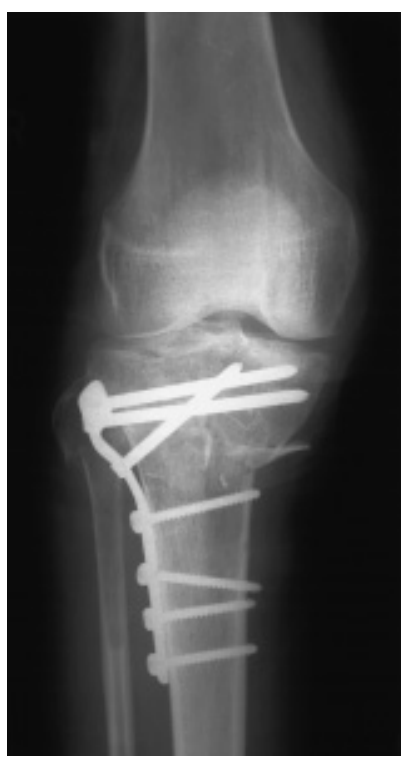

Abb. 2 Röntgenbild Hochformat.

Die Planung des weiteren Vorgehens zur Korrektur ist abhängig von der initialen Frakturgeometrie oder der Gelenkachsenabweichung, den hierdurch zu erwartenden Folgen und von der Art der durchgeführten Osteosynthese selbst, wobei patientenspezifische Gegebenheiten und die Compliance ebenso berücksichtigt werden müssen. 


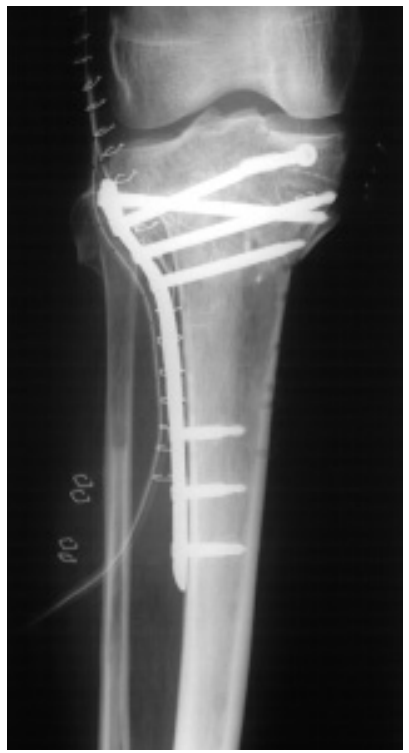

Abb. 3 Röntgenbild Hochformat.

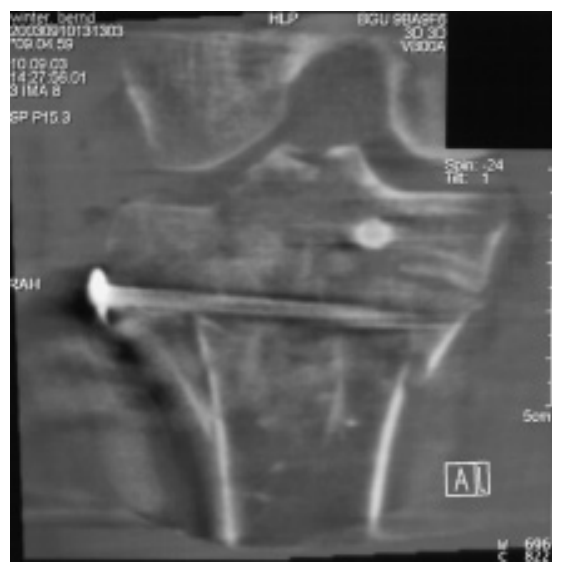

Abb. 4 Röntgenbild Querformat.

Die Art des Eingriffes sowie der nächst mögliche und sinnvolle Zeitpunkt der Korrektur muss unter Beachtung der allgemein gültigen Gesetzmäßigkeiten der Wundheilung und der Knochenbruchheilung geplant werden.

\section{Lösungsmöglichekeiten}

Bei Tibiakopfbrüchen kann man extraartikuläre Frakturen von partiell- oder vollständig artikulären Frakturen unterscheiden, wobei es sich in der Mehrzahl der Fälle um Brüche mit Gelenkbeteiligung handelt. In diesen Fällen ist primär das Rekonstruktionsergebnis der Gelenkfläche und sekundär die Gelenkachsenstellung für die weitere Prognose und Entwicklung einer posttraumatischen Arthrose ausschlaggebend.

Für den Operateur stellt sich die Frage, ob das augenblickliche Ergebnis durch chi- rurgische Mittel zu verbessern ist und durch welche korrigierenden Maßnahmen diese Verbesserung erreicht werden kann. Nach Reposition-, Rekonstruktionoder Achsenkorrektur muss eine erneute Osteosynthese durchgeführt werden. Zur Stabilisierung kann ein Verfahrenswechsel oder die Erweiterung der bestehenden Fixierung durchgeführt werden.

\section{Lösungsmöglichkeit bei verbleibender Instabilität}

\section{Beispiel 2}

63-jähriger Patient mit einem vollständig artikulären mehrfragmentären Tibiaplateau und einer komplexen irregulären proximalen Tibiaschaftfraktur (41C1) 42C1).

Die Rekonstruktion der Gelenkfläche erfolgte mittels Schrauben und einer langstreckigen winkelstabilen Osteosynthese der prox. Tibia. Zwei Monate nach operativer Versorgung zeigt sich aufgrund einer verbleibenden Instabilität eine verzögerte Frakturheilung. Eine zusätzlich ventrale Klammer brachte die Fraktur bei jetzt ausreichender Stabilität zur Ausheilung (Abb.5).

Eine Osteosynthese führt nur bei ausreichender Stabilität der Fragmente zur Frakturkonsolidierung, wobei nur Mikrobewegungen die Konsolidierung fördern.

Bei Repositions- und Rekonstruktionsmaßnahmen ist der Zeitpunkt der Reoperation, abhängig von den Wundverhältnissen so früh wie möglich (innerhalb der nächsten zwei Wochen) zu wählen, damit nicht die Knochenbruchheilung das exakte Zusammenfügen der Bruchstücke erschwert.

Lösungsmöglichkeit bei ungenügender Reposition des Tibiaplateaus und frühzeitiger Diagnostik

\section{Beispiel 3}

30-jähriger Patient mit einer vollständig artikulären mehrfragmentären medialen und lateralen Fraktur (C3.3). Es erfolgte die Rekonstruktion der Gelenkfläche mittels Schraubenosteosynthese und die Überbrückung der metaphysären und proximalen Schaftfraktur mit einem Hybridfixateur, um Übungsstabilität zu erreichen (Abb.2). Die postoperative computertomographische Kontrolle des Repositionsergebnisses zeigte deutliche

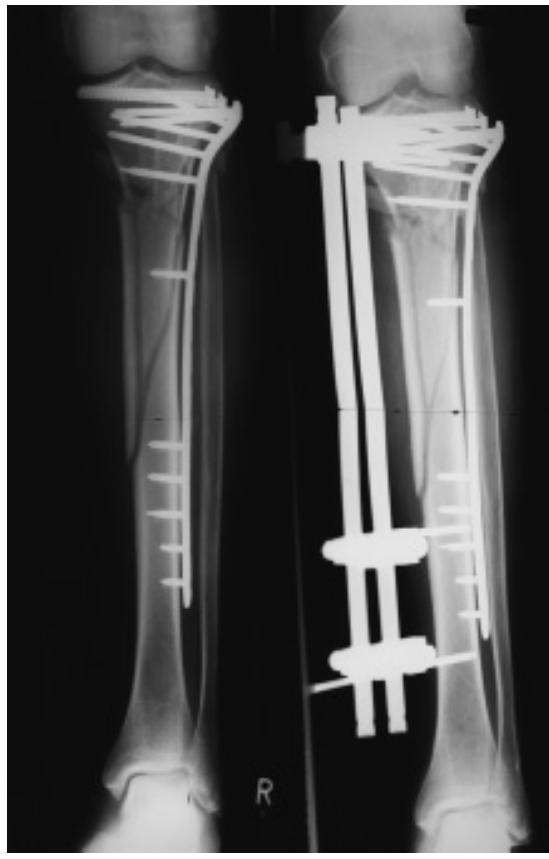

Abb.5 Röntgenbild Querformat.

verbliebene korrekturbedürftige Inkongruenzen des Tibiaplateaus.

Hierbei wurde ein Verfahrenswechsel auf eine LC-Winkelplatte nach erneuter offener Reposition und Rekonstruktion der Gelenkfläche durchgeführt (Abb.2). Durch den jetzt möglichen Verzicht auf den Fixateur externe wurde gleichzeitig bei besserer Übungsstabilität der Patientenkomfort gesteigert.

Lösungsmöglichkeit bei ungenügender Reposition des Tibiaplateaus und verzögerte Diagnostik

Beispiel 4

29-jährige Patientin mit einer partiell artikulären Spalt-impressions-Fraktur (B3). Auswärtig wurde eine L-Plattenosteosynthese zur Rekonstruktion des Tibiakopfes durchgeführt. Die radiologische Diagnostik 5 Monate nach operativer Versorgung zeigte einen verbliebenen ausgeprägten Defekt mit intraartikulärer Schraubenlage (Abb. 7/8). Bei der massiven arthroskopisch bestätigten Zerstörung des lat. Tibiaplateaus kann nur der Versuch unternommen werden, die posttraumatische Arthrose hinauszuzögern und Zeit bis zur Versteifung des Kniegelenkes oder der Prothesenimplantation zu gewinnen. Zur Rekonstruktion des Tibiaplateaus wurde ein periostüberzogener trikortikaler Beckenkammblock eingesetzt (Abb.9 und 10). 


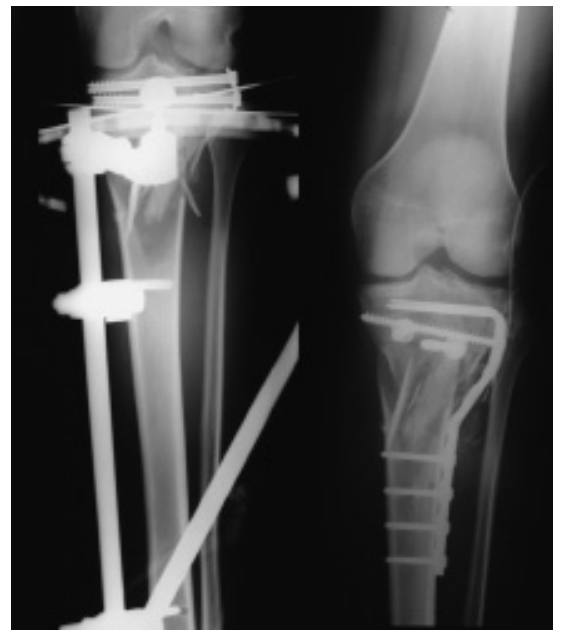

Abb. 6 Röntgenbild Querformat.

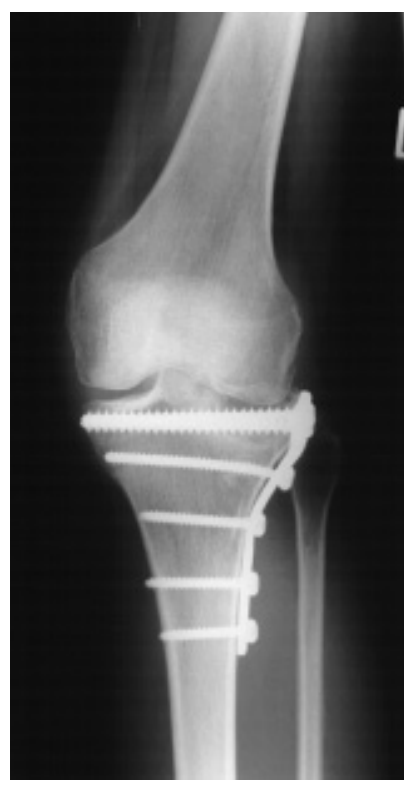

Abb. 7 Röntgenbild Hochformat.

Die frühzeitige Diagnostik des Rekonstruktionsverlustes der Gelenkfläche ist ein Ausschlag gebender Faktor für die weitere Prognose des Kniegelenkes.

Bei guter Rekonstruktion der Gelenkfläche aber zunehmender Achsenabweichungen des Tibiaplateaus sollten Korrekturen erst nach ausreichender knöcherner Konsolidierung der Gelenkbrüche durchgeführt werden.

Lösungsmöglichkeit bei

Achsenabweichung des Tibiaplateaus

Beispiel 5 und 6

Bsp. 5.: 53-jähriger Patient mit einer vollständig artikulären Trümmerfraktur des

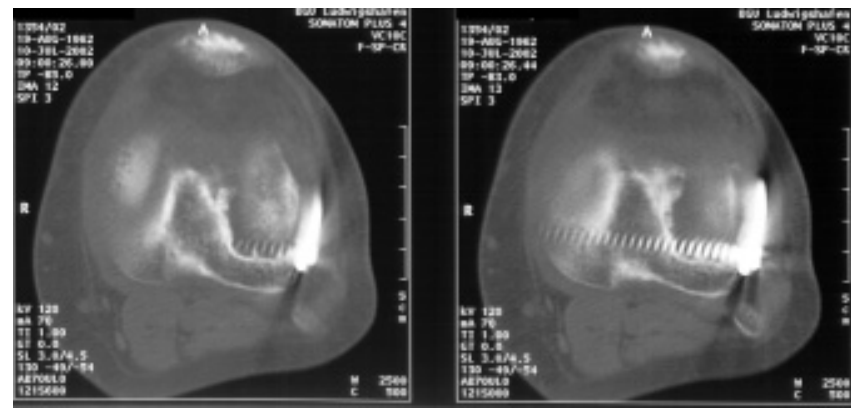

Abb. 8 Röntgenbild Querformat.

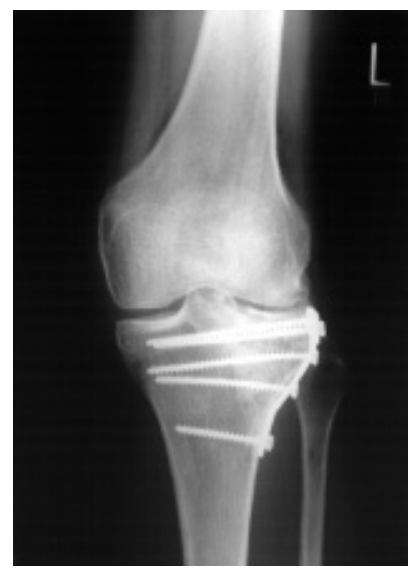

Abb. 9 Röntgenbild Hochformat.

lateralen Tibiaplateaus (C1.1). Es wurde eine Schraubenosteosynthese durchgeführt. Die Ausheilung erfolgte unter einer Achsenabweichung von $10^{\circ}$ varus ( $O$-BeinStellung) (Abb.11 und 12). Arthroskopisch bestätigt sich im Verlauf eine mediale Gonarthrose, weswegen eine Umstellungsosteotomie nach Metallentfernung der Schrauben erfolgte (Abb.13 und 14).

Bsp. 6.: 23-jährige Patientin mit einer Zertrümmerung des lateralen Tibiaplateaus (C1.1). Es wurde eine L-Plattenrekonstruktion durchgeführt. Die Konsolidierung der Fraktur erfolgt unter Achsenabweichung mit $10^{\circ}$ Varusstellung. Unter Berücksichtigung des Alters der Patientin wurde eine präventive valgisierende Umstellungsosteotomie durch das Einbolzen eines Beckenkammblocks bei einliegen- der lateraler Osteosynthese durchgeführt (Abb.15).

Neben dem Ausmaß der Gelenkachsenabweichung sind zur Indikationsstellung einer Umstellungsosteotomie zwingend die spezifischen Gegebenheiten der Patienten zu berücksichtigen.

Ein weit gehend knöchern konsolidiertes Tibiaplateau ist auch Voraussetzung für die Implantation einer Gelenkprothese bei der frühen posttraumatischen Gonarthrose (siehe auch Bsp. 7).

\section{Lösung frühposttraumatischer} Gonarthrose nach Tibiakopffraktur

Beispiel 7

53-jähriger Patient mit einer partiell lateralen Spalt-Impressionsfraktur (B3.1). Die Rekonstruktion und Stabilisierung des Tibiaplateaus erfolgte mit einer L-Platte. Im weiteren Verlauf entwickelte sich eine Teilnekrose des lat. Tibiaplateaus mit folgender Gonarthrose. 9 Monate nach osteosynthetischer Versorgung der Tibiakopffraktur wurde daraufhin eine zementierte, computernavigierte KTP implantiert (Abb.16).

Die Implantation einer KTP nach einer fehlgeschlagenen Osteosynthese des Tibiakopfes z. B. eine Umstellungsosteotomie ist erheblich schwieriger als eine primäre KTP-Implantation bei einer Gonarthrose.

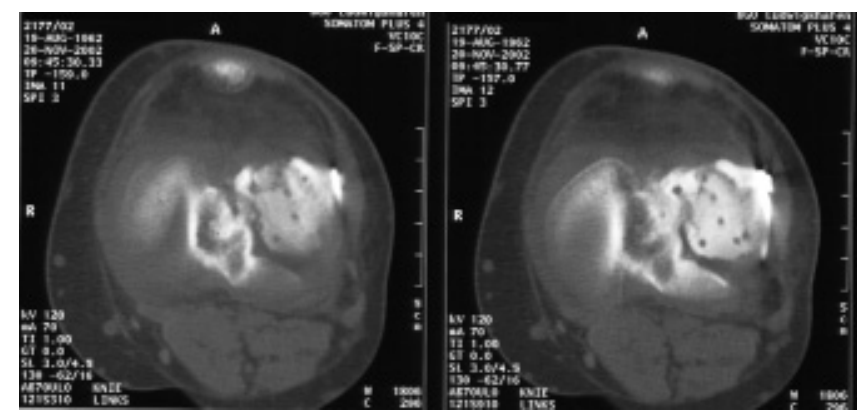

Abb.10 Röntgenbild Querformat. 
11,12
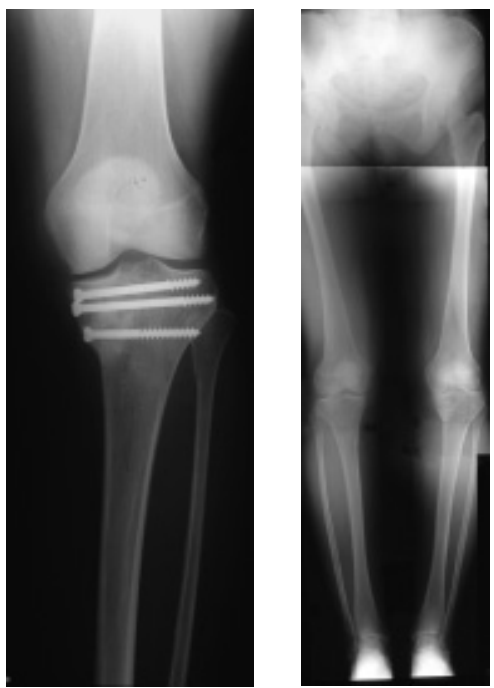

Abb.11 Röntgenbild Hochformat. Abb.12 Röntgenbild Hochformat.

13,14
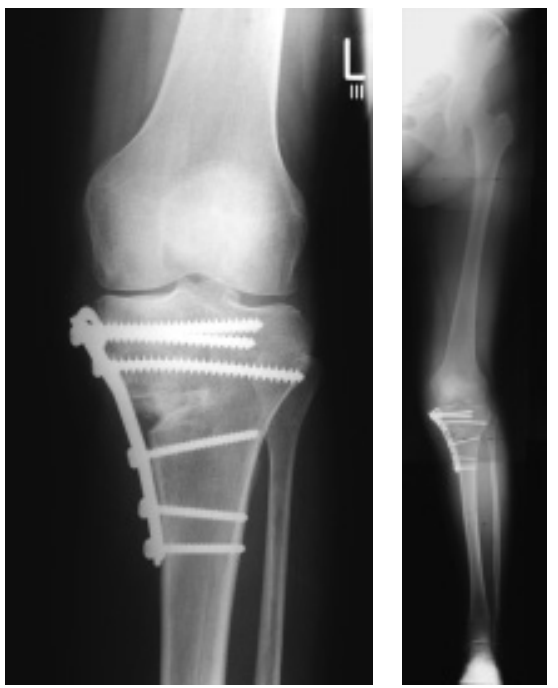

Abb.13 Röntgenbild Hochformat. Abb.14 Röntgenbild Hochformat.

\section{Schlussfolgerung}

Eine fehlgeschlagene Osteosynthese des Tibiakopfes ist sorgfältig zu analysieren, wobei sich die Lösungsmöglichkeiten aus dem zu behebenden Fehler wie Fehlstellung und oder Achsenabweichung, aber auch dem Zeitpunkt nach der Versorgung richten.

Für die korrigierende Versorgung gilt uneingeschränkt das Prinzip der Gelenkflächenrekonstruktion und Übungsstabilität. Patientenspezifische Gegebenheiten wie z.B. die Compliance, das Alter, die Mobilität und das soziale Umfeld sind hierbei genauso zu berücksichtigen wie der Zeitpunkt des Eingreifens. Der Zeit-

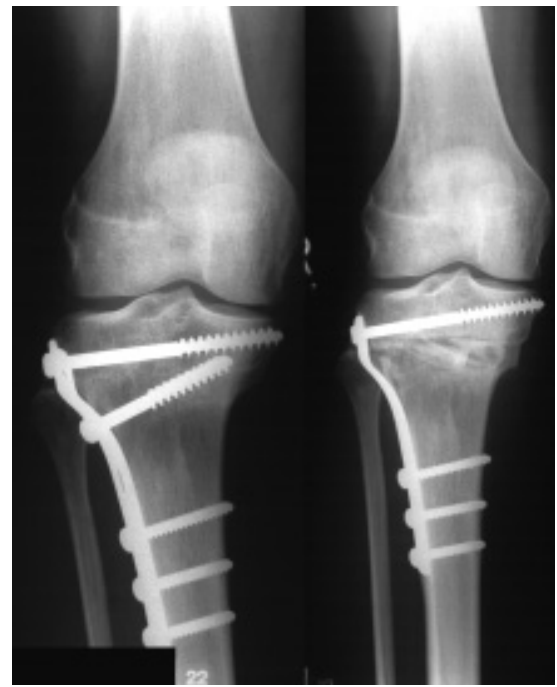

Abb.15 Röntgenbild Querformat.

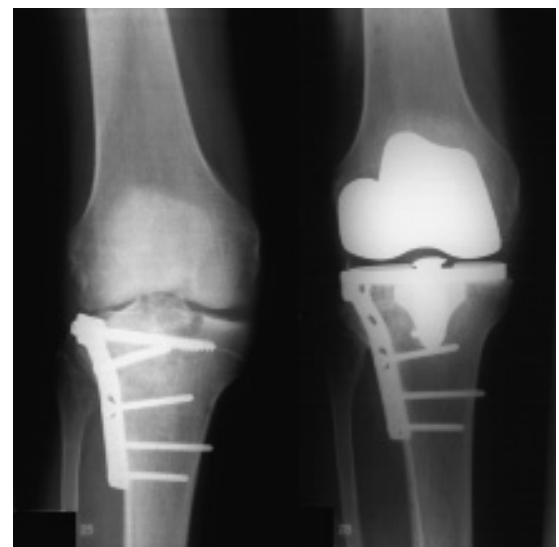

Abb.16 Röntgenbild Querformat.

punkt der Reoperation, abhängig von den Wundverhältnissen, ist so früh wie möglich (innerhalb der nächsten zwei Wochen) zu wählen, damit nicht die fortschreitende Bruchheilung das exakte Zusammenfügen der Bruchstücke erschwert. Bei zunehmender Achsenabweichung des Tibiaplateaus sollten Korrekturen erst nach ausreichender knöcherner Konsolidierung der Gelenkbrüche durchgeführt werden.

Gelenkflächenrekonstruktionen nach Wochen bis Monaten sind außerordentlich schwierig und unter Umständen nur durch die Implantation eines Kunstgelenkes oder die Kniegelenksarthrodese zu lösen.

\section{Literatur}

1 Ali AM, El-Shafie M, Willett KM. Failure of Fixation of Tibial Plateau Fractures. Journal of Orthopaedic Trauma. May 2002; 16 (5): $323-329$

2 Harris TE, Ruth JT. Operative management of fractures of the tibial plateau. Am J Knee Surg 2001 Spring; 14 (2): 129-34. Review

3 Hertel P. Tibial plateau fractures. Unfallchirurg $1997 \mathrm{Jul} ; 100$ (7): $508-23$

4 Kotter A, Ruter Aa. Fractures of the tibial plateau. Unfallchirurg 1997 Sep; 100 (9): 742 -9

5 Zatti G, Bini A, Surace MF, Cerubino P. The surgical treatment of fractures of the proximal end of the tibia: a review of cases as related to prognostic factors. Chir Organi Mov 2000 Oct-Dec; 85 (4): $371-80$

\section{Dr. med. Thomas Braun}

Assistenzarzt Unfallchirurgie

Prof. Dr. med. Andreas Wentzensen

Ärztlicher Direktor

BG-Unfallklinik

Ludwig-Guttmann-Str. 13

D-67071 Ludwigshafen 